\title{
A REMARK ON THE MAXIMAL DILATATION OF A QUASICONFORMAL MAPPING
}

\author{
MATTI VUORINEN
}

\begin{abstract}
A lower bound for the maximal dilatation of a quasiconformal self-mapping of a uniform domain $G$ in $R^{n}$ with connected boundary is proved provided that the boundary is kept pointwise fixed by the mapping and that a prescribed point $a$ is mapped on another point $b$ in $G$. The lower bound is given in terms of the quasihyperbolic distance from $a$ to $b$.
\end{abstract}

1. Introduction. Let $G$ be a proper subdomain of $R^{n}(n \geq 2)$, let $a, b \in G$ be given, and let $f: \bar{G} \rightarrow \bar{G}$ be a quasiconformal (qc) homeomorphism such that $f(a)=b$ and $f(x)=x$ for $x \in \partial G$. In this note we shall give a lower bound for the maximal dilatation $K(f)$ of $f$.

Gehring and Palka $[\mathbf{3}]$ have proved that there exists a qc mapping $g: \bar{G} \rightarrow \bar{G}$ with the above properties such that

$$
\log K(g) \leq 2(n-1) k_{G}(a, b),
$$

where $k_{G}(a, b)$ is the quasihyperbolic distance $([\mathbf{3}])$ from $a$ to $b$. Here the following lower bound will be established

$$
K(f) \geq c(n, G) k_{G}(a, b)^{n}
$$

where $c(n, G)$ is a positive number depending only on $n$ and $G$. The estimate (1.2) is valid under the following two assumptions (i) $G$ is a uniform domain with connected boundary; (ii) $k_{G}(a, b)$ exceeds a bound, depending on the parameters in (i). Uniform domains were introduced by Martio and Sarvas [4] and have been studied by Gehring and Osgood [2].

For the dimension $n=2$ and $G=$ the unit disc, the exact bound in (1.2) has been determined by Teichmüller [5]. An $n$-dimensional counterpart of Teichmüller's result under an additional symmetry assumption was obtained by Anderson and Vamanamurthy $[\mathbf{1}]$. The proof of $(1.2)$ is based on a characterization of uniform domains due to Gehring and Osgood [2]. I am grateful to Professor F. W. Gehring for bringing this problem to my attention, to Professor G. D. Anderson and Professor J. Sarvas for conversations, and to the referee for some remarks.

2. Quasihyperbolic metric. Let $G \subset R^{n}$ be a domain, $G \neq R^{n}$, and let $d(x)=\operatorname{dist}(x, \partial G) \in(0, \infty)$ for $x \in G$. The quasihyperbolic distance between $a$ and $b$ in $G$ is defined by (cf. [3])

$$
k_{G}(a, b)=\inf _{\gamma} \int_{\gamma} d(x)^{-1} d s
$$

Received by the editors April 5, 1982 and, in revised form, November 28, 1983.

1980 Mathematics Subject Classification. Primary 30C60.

Key words and phrases. Quasiconformal mappings. 
where $\gamma$ runs through all rectifiable curves in $G$ with $a, b \in \gamma$. It was proved by Gehring and Palka $[\mathbf{3}]$ that $k_{G}$ is a metric in $G$, which defines the Euclidean topology of $G$. Several other properties of $k_{G}$ can be found in [3 and 4].

A domain $G \subset R^{n}$ is said to be uniform (cf. $\left.[2,4]\right)$ if there exist constants $\tilde{A}$ and $\tilde{B}$ such that each pair of points $x, y \in G$ can be joined by a rectifiable $\operatorname{arc} \gamma \subset G$ for which

$$
\left\{\begin{array}{l}
s(\gamma) \leq \tilde{A}|x-y| \\
\min \left\{s\left(\gamma_{x}\right), s\left(\gamma_{y}\right)\right\} \leq \tilde{B} d(z) \quad \text { for all } z \in \gamma
\end{array}\right.
$$

where $s(\gamma)$ denotes the Euclidean length of $\gamma$ and $\gamma_{x}\left(\gamma_{y}\right)$ denotes the part of $\gamma$ between $z$ and $x$ (resp. $z$ and $y$ ).

Next define $j_{G}(x, y)=\log (1+|x-y| / \min \{d(x), d(y)\})$ for $x, y \in G$.

The following characterization of uniform domains is due to Gehring and Osgood. Because our definition of $j_{G}(x, y)$ differs slightly from the corresponding definition in $[2]$ the numerical values of the costants $c$ and $d$ may be slightly different from those in $[\mathbf{2}]$.

2.1. LEMma. A domain $G$ is uniform if and only if there are constants $c$ and $d$ such that $k_{G}(x, y) \leq c j_{G}(x, y)+d$ for all $x, y \in G$.

2.2. REMARK. If $G$ is uniform, then $k_{G}(x, y) \leq B j_{G}(x, y)$ for all $x, y \in G$ where $B \leq 3(c+d)$ as shown in [7]. A similar observation has been independently made by K. Astala and F. W. Gehring (unpublished).

For the theory of quasiconformal mappings and for the properties of the modulus of a curve family the reader is referred to Väisälä's book [6]. For $x \in R^{n}$ and $r>0$ let $B^{n}(x, r)=\left\{z \in R^{n}:|z-x|<r\right\}$ and $S^{n-1}(x, r)=\partial B^{n}(x, r)$.

3. The result. We first prove a lemma. The boundary of a domain in $R^{n}$ is taken in the topology of $\bar{R}^{n}=R^{n} \cup\{\infty\}$.

3.1. LEMMA. Let $G$ be a domain in $R^{n}$ with connected boundary $\partial G$, let $a, b \in$ $G$, and let $x \in \partial G$ be such that $d(a)=|a-x| \leq|b-x|$. For every homeomorphism $f: \bar{G} \rightarrow \bar{G}$ with $f(a)=b$ and $f|\partial G=i d| \partial G$, the following lower bound holds

$$
K(f) \geq d_{n}\left(\log \frac{|b-x|}{|a-x|}\right)^{n}
$$

where $d_{n}$ is a positive number depending only on $n$.

Proof. Write $m=|a-x|$ and $M=|b-x|$. Then the segment $[a, x]=$ $\{a u+x(1-u): 0 \leq u \leq 1\} \subset G \cup\{x\}$. We may assume $M>m$. Fix $t \in(m, M)$. Write $A=\partial G \backslash \bar{B}^{n}(x, t)$ and let $\Gamma_{t}=\Delta([a, x], A ; G)$ be the family of all curves in $G$ joining $[a, x]$ to $A[6$, p. 21]. Since $f|\partial G=i d| \partial G$ it follows that $f A=A$ and further because $f(a)=b$ and $\partial G$ is connected

$$
S^{n-1}(x, r) \cap f[a, x] \neq \varnothing, \quad S^{n-1}(x, r) \cap f A \neq \varnothing,
$$

for $r \in(t, M)$. From $(3.2)$ and $[6,7.5,10.12,13.1]$ it follows that

$$
c_{n} \log \frac{M}{t} \leq M\left(f \Gamma_{t}\right) \leq K(f) M\left(\Gamma_{t}\right) \leq K(f) \omega_{n-1}\left(\log \frac{t}{m}\right)^{1-n}
$$


where $c_{n}$ and $\omega_{n-1}$ are positive numbers depending only on $n$. Since $t \in(m, M)$ is arbitrary, we get

$$
K(f) \geq \frac{c_{n}}{\omega_{n-1}} \sup \{h(t): t \in[m, M]\} ; \quad h(t)=\left(\log \frac{M}{t}\right)\left(\log \frac{t}{m}\right)^{n-1} .
$$

Set $t=m^{p} M^{1-p}, p \in(0,1)$. Then

$$
h\left(m^{p} M^{1-p}\right)=p(1-p)^{n-1}\left(\log \frac{M}{m}\right)^{n} .
$$

For $p=1 / n$ we get the desired lower bound

$$
K(f) \geq d_{n}\left(\log \frac{M}{m}\right)^{n} ; \quad d_{n}=c_{n}(n-1)^{n-1} /\left(\omega_{n-1} n^{n}\right) .
$$

3.3. THEOREM. Let $G$ be a uniform domain in $R^{n}$ with connected boundary $\partial G$, and let $a, b \in G$. Then there exists a positive constant $c(n, G)$ depending only on $n$ and $G$ such that for every homeomorphism $f: \bar{G} \rightarrow \bar{G}$ with $f(a)=b$ and $f|\partial G=i d| \partial G$

$$
K(f) \geq c(n, G) k_{G}(a, b)^{n}
$$

whenever $k_{G}(a, b)$ exceeds a bound, depending only on $n$ and $G$.

Proof. We may assume $d(a) \leq d(b)$. Fix $z \in \partial G$ such that $d(a)=|a-z|$. Then we have

$$
\frac{|b-z|}{|a-z|}=\frac{|b-z|}{d(a)} \geq \frac{|a-b|}{|a-z|+|b-z|} \frac{|b-z|}{d(a)} \geq \frac{1}{2} \frac{|a-b|}{d(a)} .
$$

Since $G$ is uniform, there exists a number $B$ such that $k_{G}(x, y) \leq B j_{G}(x, y)$ for all $x, y \in G$ (cf. Remark 2.2). Since $d(a) \leq d(b)$, we get by the definition of $j_{G}$

$$
\frac{|a-b|}{d(a)} \geq \exp \left(k_{G}(a, b) / B\right)-1 \geq k_{G}(a, b) / B \text {. }
$$

Assume now that $k_{G}(a, b) \geq 2 B$. For $k_{G}(a, b) \geq 2 B$ we can improve the last inequality as follows

$$
\frac{|a-b|}{d(a)} \geq \exp \left(k_{G}(a, b) / B\right)-1 \geq 2 \exp \left(k_{G}(a, b) /(2 B)\right) .
$$

This inequality, together with 3.1 and (3.4) yields

$$
K(f) \geq c(n, G) k_{G}(a, b)^{n} ; \quad c(n, G)=d_{n}(2 B)^{-n}
$$

for $k_{G}(a, b) \geq 2 B$, as desired.

3.5. REMARK. It is an interesting question, whether the best possible lower bound for $K(f)$ in 3.3 is greater than one whenever $a \neq b$. We now show that this is not true when $n=3$ and $G=R^{3} \backslash Z, Z=\{(0,0, z): z \in R\}$. Let $a=(1,0,0)$ and $b=-a$, and let $f$ be a rotation around the $z$-axis with $f(a)=b$ such that $f$ keeps the $z$-axis $Z$ pointwise fixed. Then $f$ is conformal and hence $K(f)=1$, while by a symmetry argument we see that $k_{G}(a, b)=\pi$. It is easy to verify that $R^{3} \backslash Z$ is uniform. 


\section{REFERENCES}

1. G. D. Anderson and M. K. Vamanamurthy, An extremal displacement mapping in n-space, Complex analysis, Joensuu, 1978, pp. 1-9; Lecture Notes in Math., vol. 747, Springer-Verlag, 1979.

2. F. W. Gehring and B. G. Osgood, Uniform domains and the quasihyperbolic metric, J. Analyse Math. 36 (1979), 5074.

3. F. W. Gehring and B. P. Palka, Quasiconformally homogeneous domains, J. Analyse Math. 30 (1976), 172-199.

4. O. Martio and J. Sarvas, Injectivity theorems in plane and space, Ann. Acad. Sci. Fenn. Ser. A I Math. 4 (1978/1979), 383-402.

5. O. Teichmüller, Ein Verschiebungssatz der quasikonformen Abbildung, Deutsche Math. 7 (1944), 336-343.

6. J. Väisälä, Lectures on n-dimensional quasiconformal mappings, Lecture Notes in Math., vol. 229, Springer-Verlag, 1971.

7. M. Vuorinen, Conformal invariants and quasiregular mappings, in preparation.

Department of Mathematics, University of Helsinki, Hallituskatu 15, SF00100 HELSINKI 10, FinLAND 